\title{
Reflection Coefficient S1 1 Related Measurement System for Label-Free Cell Seeding Analysis and Drug Testing in a Three-Dimensional (3D) Cell Culture Model
}

Lornejad-Schäfer MR ${ }^{1}$, Hilber $\mathbf{W}^{2}$ and Schäfer $\mathbf{C}^{1 *}$

${ }^{1}$ BioMed-zet Life Science GmbH, Industriezeile 36/VII, 4020 Linz, Austria

2Institute for Microelectronics and Microsensors, Johannes Kepler University Linz, Altenbergerstrasse 69, 4040 Linz, Austria

\begin{abstract}
Context: With regard to two-dimensional (2D) cell cultures in flat dishes, most of the three-dimensional (3D) cell cultures have advantages and gain importance in life sciences. But, their higher complexity requires adaptation of existing methods or different measurement systems in order to achieve accurate results. Bio-Impedance spectroscopy (Bio-IS) is a label- free and non-destructive method that is considered for analysis of 3D cell models.

Purpose: We design a new Bio-IS measurement system for a 3D liver cell model to assess cell seeding and drug effects.

Experiment Methods: We measured reflection coefficient S11 with differently (disc- and needle-like) shaped electrodes, which are in defined contact with the 3D liver cell model, metabolic activity (MTT test), and cytotoxicity (LDH assay). Results: We demonstrate that reflection coefficient S11 may be utilized to assess the 3D liver cell system in the frequency range of $\beta$-dispersion at $73 \mathrm{MHz}$ only by means of the needle-like electrodes. This enables a reliable label-free and non-destructive determination of cell seeding and drug effects in the 3D liver cell culture model. The physical results have been verified by destructive biochemical methods (LDH and MTT assay).
\end{abstract}

Conclusion: Our Bio-IS system for 3D liver cell models using needle-like electrodes enables label-ree and nondestructive determination of cell seeding and drug effects.

Keywords: Impedance; Label-free method; Non-invasive; 3D cell culture model; Drug testing

Abbreviations: Acetaminophen (APAP) or N-acetyl-p-
aminophenol or Paracetamol DMSO: Dimethyl Sulfoxide; Bio-IS:
Bio-Impedance Spectroscopy; f: Frequency; FBS: Fetal Bovine Serum;
LDH: Lactate Dehydrogenase; MTT: 3-(4,5-dimethylthiazol-2-yl)-
2,5-diphenyltetrazolium bromide PBS: Phosphate Buffer Saline; PS:
Polystyrene; 3D: Three Dimensional

\section{Introduction}

Bio-IS measures the electrical properties of a biological test object when current flows through it. It is an emerging tool for biomedical research and medical diagnosis with a broad range of applications. For example, Bio-IS is used to estimate body composition, determine total body water levels and calculate the fat/fat-free mass [1]. Bio-IS can be used for tissue classification, as in breast cancer screening [2]. On the cellular level, Bio-IS is used in commercial blood analyzers to determine hematocrit levels [3]. Various cell-based impedimetric sensing systems are currently commercially available [4-6]. Several other devices and systems that study cell-substrate interactions in vitro have been reported in scientific literature [7].

The application spectrum of Bio-IS is expanding towards advanced cell culture techniques and complex cell systems that are used for tissue engineering and pharmacological and toxicological in-vitro testing. This analysis technique is expected to provide informative results about tissue structure, physiological states, and cell functions.

Determination and counting of cells or analysis of cell properties and cell behavior as a response to a drug in $3 \mathrm{D}$ cell culture models is a challenge. Established analytical methods for this purpose fail or can only partially be transferred from conventional $2 \mathrm{D}$ cell cultures in petri dishes [8]. For instance, when using disc-like polystyrene scaffolds, it is difficult to view and determine seeded cells using the light microscope because towards $3 \mathrm{D}$ direction extended material is $1 \mathrm{~mm}$ thick and often not translucent. In addition, the conventional cell detachment and digestion procedure for counting cells is more difficult and will not always give reliable results.

Some cells may stay attached in scaffold niches after trypsinization and may undergo prolonged digestion. An attempt to detach all cells from the carrier material may destroy and resolve cells.

The use of sophisticated valuable cells, stem cells or progenitor cells, such as HepaRG cells coming from human liver, in a 3D cell culture model or/and in bioreactors makes it necessary to follow long proliferating or/and differentiation protocols that bear the risk of contaminating cell culture, and may produce non-replicable effects, which increases working time and costs.

For advanced cell cultures, such as $3 \mathrm{D}$ cell culture, it is important to monitor cell viability, proliferation, differentiation states or contaminations, the best time to start an experiment, meanwhile and after its termination. In contrast, these evaluations can be easily performed in conventional, label- free 2D cell culture models, because the cells are generally visible by means of a light microscope.

*Corresponding author: Christine Schäfer, BioMed-zet Life Science $\mathrm{GmbH}$ Industriezeile 36/I, 4020 Linz, Austria, Tel: (732) 770325-250; E-mail: chrschaefer9@googlemail.com

Received February 11, 2014; Accepted April 16, 2014; Published April 22, 2014

Citation: Lornejad-Schäfer MR, Hilber W, Schäfer C (2014) Reflection Coefficient S11 Related Measurement System for Label-Free Cell Seeding Analysis and Drug Testing in a Three-Dimensional (3D) Cell Culture Model. J Biosens Bioelectron 5: 151. doi: 10.4172/2155-6210.1000151

Copyright: @ 2014 Lornejad-Schäfer MR, et al. This is an open-access article distributed under the terms of the Creative Commons Attribution License, which permits unrestricted use, distribution, and reproduction in any medium, provided the original author and source are credited. 
The aim of this study was to explore the application of Bio-IS in a human 3D liver cell culture for label-free and non-destructive determination of cell seeding and drug effects. Therefore, differently shaped electrodes were designed and tested for their applicability and it was assessed whether the Bio-IS at specific frequencies can be linked to cell seeding or drug effects in the human $3 \mathrm{D}$ liver cell culture. We investigated APAP, an over-the-counter drug, that is considered a safe drug, but is often abused or overdosed and many hepatotoxic APAP effects on cellular level have been enlightened [9]. Therefore, APAP is a reference substance for hepatotoxicity. Following toxic doses of APAP, intracellular glutathione pools are depleted, leading to oxidative damage and binding of reactive metabolites covalently to liver proteins. Subsequently, mitochondrial dysfunction leads to necrosis [9].

\section{Material and Methods}

Materials for electrode construction were provided from the Institute for Microelectronics and Microsensors (JKU, Linz, Austria). Materials for cell culture were obtained from Greiner Bio-one (Austria) and Lonza (Belgium). Cell culture media and all other chemicals were purchased from Sigma-Aldrich (Austria). The Cytotoxicity Detection Kit (LDH) was obtained from Roche Diagnostics (Germany). The polystyrene (PS) scaffolds were purchased from Gesellschaft für Werkstoffprüfung mbH (GWP), Zorneding, Germany.

\section{D Human liver cell model}

Human in vitro models such as human liver 3D cell models give rise to more reliable species-specific testing results, are easier and cheaper to perform and are ethically better accepted than animal experiments [10]. HepaRG cells were obtained from BioPredic (France) and were grown and kept in a permanent proliferating state in conventional culture flasks (Greiner-bio one) in a humidified atmosphere at $37^{\circ} \mathrm{C}$ and $5 \% \mathrm{CO} 2$. The HepaRG cells grow adherently on the flask bottom (2D cell culture).

Cells were detached from the surface using trypsin and separation through pipetting up and down, and the cell suspension was centrifuged. After discarding the supernatant, the cell pellet was diluted with fresh medium. An aliquot was used to determine the cell number using CASY ${ }^{\circledast}$ Cell counter [11].

To construct the liver $3 \mathrm{D}$ cell model, $1 \times 10^{6} \mathrm{HepaRG}$ cells were seeded on porous plasma activated PS scaffolds and cultivated in Williams' E medium supplemented with $10 \%$ Fetal Bovine Serum (FBS), 100 units $/ \mathrm{ml}$ penicillin, $100 \mu \mathrm{g} / \mathrm{ml}$ streptomycin, $5 \mu \mathrm{g} / \mathrm{ml}$ insulin, $2 \mathrm{mM}$ glutamine, and $0.5 \mu \mathrm{M}$ hydrocortisone hemisuccinate. Differentiation of the HepaRG cells was induced after a one week proliferating period with a $1 \%$ DMSO- containing medium and took three weeks.

\section{Electrical properties of cells and tissues}

Electrical Impedance Spectroscopy (IS) of a test object is performed by measuring the complex electrical impedance $(\mathrm{Z})$ and its phase angle $(\Phi)$ at different frequency points within a specified frequency band, from the measured potentials (V) developed by injecting a constant sinusoidal current signal (I) to the object [12].

Bio-IS measures the passive electrical properties of complex tissues or single cells. Parameters that influence the bio-impedance of a tissue or a cell cluster are the resistance of the extracellular medium and the Intracellular cytoplasma, and the resistance and capacitance of the cellular membrane. The extracellular space acts as a conductive medium. This can be described by a simplified electrical circuit $[13,14]$. In the simplest case, single cells may be modeled as a group of the three electronic components, comprising two resistances representing the intra- and extracellular conduction, and a cell- membrane capacitance [15].

Cells possess specific dielectric properties. Three different frequency-dependent main dispersions can be classified, termed $\alpha$, $\beta$ and $\gamma$ dispersions. $\alpha$ dispersion, the low- frequency dielectric cell behavior up to $10 \mathrm{kHz}$ is assumed to be dominated by current flow through membrane ion channels and counter-ion polarization along cell membranes. $\beta$-dispersion $(10 \mathrm{kHz}$ to $100 \mathrm{MHz})$ is associated with the dielectric properties of the cytoplasm-membrane interface, intracellular membrane structures, and the polarization of proteins and other organic macromolecules. In this frequency range, membrane capacitance becomes electrically conducting: it short-circuits the membrane resistance, and the electric field begins to penetrate the interior of the cell. $\gamma$-dispersion $(100 \mathrm{MHz}$ to $100 \mathrm{GHz})$ is determined by the polarization of free and bound water molecules, protein-protein interactions, and the dissociation/association relaxation of small charged groups [15].

\section{Reflection coefficient S11 measurement}

Bio-impedance is assessed by measuring the reflection coefficient $\mathrm{S} 11(\mathrm{~dB})$, defined as $\mathrm{S} 11=(\mathrm{Z}-\mathrm{Z} 0) /(\mathrm{Z}+\mathrm{Z} 0)$, where $\mathrm{Z} 0$ and $\mathrm{Z}$ denote the characteristic line impedance and impedance of the sensor, respectively.

The dielectric properties of the 3D liver cell model between two electrodes (Figure 1) are measured by emitting electromagnetic waves, and measuring amplitude and phase of the reflected wave. In this configuration, the cells of the 3D liver cell model act as a network of resistors and capacitors, which affect the recorded reflection coefficient S11.

Reflection coefficient S11 measurements were done with a commercial network analyzer (Agilent E5061B, Agilent Technologies Inc., Palo Alto, CA, USA), which allows the analysis of the reflection coefficient in the frequency range between $5 \mathrm{~Hz}$ and $3 \mathrm{GHz}$. The two electrodes were connected to the network analyzer through a coaxial wire. The experimental setup with needle-like electrodes is shown in Figure1B.

After the reflection coefficient S11 measurement and data analysis, impedance was calculated at $73 \mathrm{MHz}$.
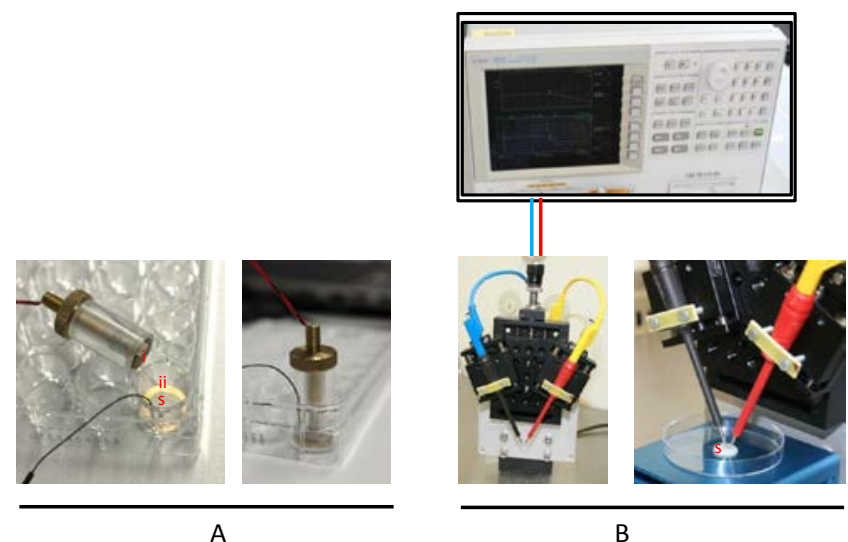

Figure 1: Electrical impedance measurement system with two different shaped electrodes. A) Disc-like electrodes. The scaffold disc is sandwiched between the ground electrode (ii) and the top electrode (i). B) Needle-like electrodes are 0.1$0.2 \mathrm{~mm}$ inserted into the scaffold disc. Distance between electrodes is $0.5 \mathrm{~cm}$; s: scaffold. 
Prior to the experiments, the measurement instrument was calibrated. For this purpose, predetermined loads were connected to the sensor (open, short and $50 \Omega$ ), thereby correcting for the influence of the cables and the electrodes on the measurement signal. In all experiments the reflection coefficient S11 was recorded in the frequency range between $5 \mathrm{~Hz}$ and $3 \mathrm{GHz}$. To measure $\mathrm{S} 11$, the $3 \mathrm{D}$ liver cell models were removed from the incubator for a short time to the sterile hood. They were taken out of the culture medium using forceps and were placed on a cell culture plate. In case of the cell number determination experiment, scaffolds without cells were used as control. In case of the analysis of drug effect, scaffolds without cells, but treated with drug were used as control.

\section{Electrode configurations}

Two electrode designs were studied: disc-like electrodes with 1 $\mathrm{cm}$ in diameter, and needle-like electrodes with adjustable distance between the electrode tips. The disc- like electrodes was covered with gold and the needle-like electrodes were covered with stainless steel to improve bio-compatibility. Figure 1 shows examples of the electrode designs.

Prior Bio-IS measurement at sterile condition under the bench, the scaffolds were transferred wet on a culture plate. The measuring apparatus was fitted with adjustable electrodes.

The disc-like electrodes match the diameter of the scaffold (Figure 1A). During the measurement, the scaffold is sandwiched between the electrodes, so that the averaged reflection coefficient $S 11(\mathrm{~dB})$ was measured over the whole scaffold.

In contrast, the needle-like electrodes mainly capture the small volume between the electrode tips (Figure 1B). To keep measuring conditions for the 3D liver cell models constant, the two needle-like electrodes were inserted into the scaffolds at a constant inter- electrode distance $(5 \mathrm{~mm})$ and penetration depth. Three measurements took place at different scaffold areas.

\section{Cell determination in porous scaffolds using reflection coefficient} S11 measurement

Liver cell cultures on polystyrene (PS) scaffolds are a new advanced 3D cell culture technique in basic science, pharmacology and tissue engineering. It promises more reliable results than conventional cell culture techniques using flat petri dishes. We have already established a human 3D liver cell model that reflected better the physiological parameters than the corresponding 2D cell culture system [16]. When adherent cells grow on the bottom of the flask (2D cell culture model), they are to a large extent in contact with the plastic material. However, when cells grow in a 3D environment, the different cell-cell and cell-matrix interactions better resemble complex tissue structures, normal proliferation, differentiation, polarization, cell behavior and intercellular interactions. The majority of $3 \mathrm{D}$ cell cultures display a more realistic biofunctionality [17]. In a cell monolayer the growth area is limited to the bottom of the culture flask, thus high cell numbers cannot be obtained, and moreover cell function is limited.

For the cell seeding experiment, the HepaRG cells, kept as proliferating cells, were seeded in the porous scaffolds and cultivated for $48 \mathrm{~h}$ with different HepaRG cell numbers with a constant seeding volume of the medium. After that, reflection coefficient S11 was measured followed by metabolic activity using MTT assay.

Determination of drug effect in the 3D human liver cell model using reflection coefficient $S 11$ measurement
After 21 days of differentiation time, the 3D liver cell models were treated with Acetaminophen (APAP), a reference substance for hepatotoxicity, for 24 hours. Reflection coefficient S11 was then measured at various APAP concentrations $(0,5,10,20 \mathrm{mM})$.

\section{Cytostaining}

The visualization of liver cells within the PS scaffolds was performed by Hematoxylin staining (Sigma Aldrich) according to the manufacturer's protocol, followed by light microscopy analysis (Zeiss Axiovert 40). Cell nuclei are stained blue.

\section{MTT assay}

Metabolic activity of all cell models was determined by reduction of MTT [18]. The MTT reagent is taken up into cells by endocytosis or protein-facilitated mechanisms. It is reduced to yield a purple formazan product that is largely impermeable to cell membranes, thus resulting in its accumulation within living cells. The samples were incubated for $2 \mathrm{~h}$ with an MTT reagent containing medium. The medium was then removed, cells were lysed and the dye was solubilized. Absorbance was quantified by measuring at $590 \mathrm{~nm}$ wavelength with a spectrophotometer (Infinite M200 Tecan, Austria). The amount of purple formazan depends on the amount of living cells in the $3 \mathrm{D}$ liver cell model. After treatment with APAP the amount of formazan produced by living cells was compared with the amount of formazan produced by the control (0 mM APAP).

Lactate Dehydrogenase (LDH) is an intracellular localized oxidoreductase which catalyses the interconversion of lactate and pyruvate. It is often used as a marker of cell and tissue breakdown, since $\mathrm{LDH}$ is a ubiquitous enzyme. $\mathrm{LDH}$ is indicative of tissue or cell damage. The release of $\mathrm{LDH}$ from cells into the medium increases when plasma membrane leakage occurs. Spectrophotometric measurements from the $3 \mathrm{D}$ liver cell model drug testing samples were performed using the LDH cytotoxicity detection kit from Roche Diagnostics according to the manufacturer's protocol. Supernatants from the cell culture samples were collected $24 \mathrm{~h}$ after APAP treatment.

\section{Results and Discussion}

The cultivation of human cells has great economic potential due to its important applications for human health, such as being a source for adult stem cells in leukemia treatment and tissue engineering, or drug discovery, and pharmacological or toxicological drug testing.

For our study, we opt for S11 measurement because it can characterize the 3D liver cell model as a "black box". The 3D liver cell model is a complex living system and at defined frequency S11 it is possible to predict its external behavior without any regard for the detailed influencing contents. The S11 measurement, using our developed apparatus, may reflect hepatic behavior to estimate cells, and pharam- and toxicological relevant doses and time points for humans health and safety. We demonstrated that reflection coefficient S11 and subsequent determination of bio- impedance $\mathrm{Z}$ is particularly interesting for the 3D liver cell model, as this is a label-free nondestructive technique that allows repeatable and low-invasive analysis. After the S11 measurement of living HepaRG cells, the cells can be kept in their original state. The 3D liver cell models can be reused for further investigation, which facilitates the study of cell growth or treatment with drugs or toxic substances over time. This will save time and minimizes costs. Especially valuable, the slowly growing human cells are increasingly used for screening, pharmacological or toxicological testing or for biomedical applications such as tissue engineering. 
The reflection coefficient S11 is a parameter originated from high frequency electronics and is used to characterize the behavior of linear electrical networks when undergoing various steady state stimuli electrical signals. Although applicable at any frequency, S- parameters are mostly used for networks operating at Radio Frequency (RF) and microwave frequencies where signal power and energy considerations are more easily quantified than currents and voltages [19]. Here, S11 $(\mathrm{dB})$ is a new relevant parameter in testing $3 \mathrm{D}$ cell culture models. As a function of frequency the dielectric properties of the $3 \mathrm{D}$ liver cell model can be measured based on the interaction of an external field with the electric dipole moment of the sample. Differences in dielectric frequency-dependent behavior can be used to determine in the 3D liver models cell numbers or alterations in cell membrane integrity or various physiological changes induced in cells following drug treatment. The dielectric properties of biological samples display extremely high dielectric constants at low frequencies, falling off in more or less distinct steps as the excitation frequency is increased. The general frequency-dependence of permittivity is referred to as dispersion. According to Schwan, there are three major dispersion regions termed $\alpha, \beta$ and $\gamma$, as discussed in section 2.2 [20].

A comparison of the resulting characteristic curve plots of reflection coefficient $\mathrm{S} 11(\mathrm{~dB})$ versus a broad range of frequencies was used by us to easily define the particular frequency where changes in the $3 \mathrm{D}$ cell culture model occur most. Furthermore, the comparison of curves helped to identify the frequency that show maximal differences in reflection coefficient $\mathrm{S} 11(\mathrm{~dB})$ values in the context of each experimental setting. At this localized frequency, we were sub sequentially able to carry out more complex analysis, such as impedance $\mathrm{Z}$ analysis.

\section{Design of electrodes for IS adapted for 3D liver cell model}

The usage of differently shaped electrodes for our study revealed that needle- like electrodes (Figure 1B) better capture the cellular changes of the 3D liver cell model than the disc-like electrodes. The disc-like electrodes (Figure 1A) that represent a coplanar waveguide structure turned out to be inappropriate, since it was practically impossible to bring them into defined contact with the 3D liver cell model. As a result, there was strong variation in the measurement signal.

During the needle-like electrodes measurement, the scaffold was penetrated by the electrodes, which means that only a small volume fraction of the cell carrier contributed to the measurement signal (Figure 1B). To proof the measurements, each 3D liver cell model was measured three times at three different points. It has been shown that the data were broadly uniform and thus it was reliable to calculate the mean values. Hence, we opted for S11 measurement by the needle-like electrode for determination of cell seeding (Figure 3 ) and drug testing (Figure 5A)

\section{D liver cell model}

The 3D liver cell model is structurally more complex due to HepaRG cells, that grow within the pores of the PS-scaffolds (Figure 2C) than the conventional cell culture where cells grow flat on the cell culture plates (Figure 2C). The unique characteristics of the HepaRG cells $[21,22]$ together with their improved biofunctionality in the scaffolds [16] make this 3D liver cell model a reliable tool for the development of new low-invasive and label-free electronic detection techniques for pharmacology and biomedicine.

\section{Determination of cell seeding in the 3D liver cell model}

Critical steps in culturing cells are the cell seeding at the beginning
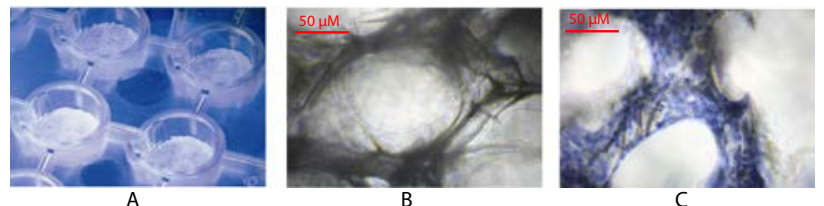

Figure 2: Polystyrene scaffolds for the cultivation of $3 \mathrm{D}$ cell model and visualization of HepaRG colonization by haematoxylin staining. A) Scaffolds are placed in custom-built 24-well culture plates. B) area of cell-less scaffold and C) HepaRG cells within a scaffold area; cell nuclei are stained blue.

and the cellular state determination prior to an experiment. Both need to be strictly controlled to avoid underproductive results. Accurate assessment of the growth of cells cultured in scaffold is a based assay. Inhomogeneous cell seeding and growth in those 3D cell models can lead to unreliable results.

To visualize cell seeding in the scaffold, we used cell nuclei staining by means of hematoxylin. Cell staining followed by light microscopy analysis allows just viewing of distinct scaffold regions of the polystyrene matrix structure (Figure $2 \mathrm{~B}$ and $2 \mathrm{C}$ ) and of the settled HepaRG cells within the scaffold. After cell nuclei have been stained blue, cell seeding was visible on the level of single cells (Figure 2C), as control an area of the unseeded scaffold did not stain blue (Figure 2B). Hematoxylin staining is a destructive method and therefore the 3D liver cell models cannot be used again.

To validate the cell seeding data assessed from reflection coefficient S11 measurement, the same 3D liver cell models were used for metabolic activity determination using MTT assay. Our results suggest that the assessment of the cell seeding at our established 3D liver cell model by means of the physical method, reflection coefficient S11, and the biochemical method, metabolic activity (MTT assay), has been achievable. We explored if seeding of different numbers of HepaRG cells into scaffolds can be determined quantitatively due to the reflection coefficient S11 (Figure 3A). Changes of the reflection coefficient S11 at the frequency range from $67-$ to- $83 \mathrm{MHz}$, where S11 value was lower than $-6 \mathrm{~dB}$, were associated with different cell numbers in the 3D liver cell models (Figure 3B). The reflection coefficient S11 profile revealed a resonance frequency of $73 \mathrm{MHz}$ at $\mathrm{S} 11=-10 \mathrm{~dB}$, where the 3D liver cell model reflection was negligible. At the frequency of $73 \mathrm{MHz}$, the lowest points were located and the curves differ mostly from the other spectra depicted at different cell numbers (Figure $3 \mathrm{~A})$. The decrease of the reflection coefficient $S 11$ values at $73 \mathrm{MHz}$ coincided with the increase of the number of cells seeded onto the scaffold (Figure 3B and 3C). In the radio frequency range cells typically exhibit a significant $\beta$-dispersion due to the Maxwell-Wagner effect at the interface between the intra- or extracellular solution and the phospholipid membrane. In addition, water molecules will cause a gamma- dispersion and any protein or macromolecule will produce dispersions at frequencies ranging from the- through the $\gamma$-dispersionrange, depending on the size and charge of the molecules. Water bound to proteins will also cause a $\delta$-dispersion [23]. $73 \mathrm{MHz}$ is primarily within the frequency range of $\beta$-dispersion. Therefore, we figure that quantitative measurements of cell numbers in the PS-scaffolds can be explained mainly through $\beta$-dispersion. $\beta$-dispersion is associated with polarization of cell membranes, proteins and other organic macromolecules [24]. The biological response to electromagnetic waves depends on one hand on the external power density, and on the other hand on the interaction with electric charges and dipoles inside of the 
A
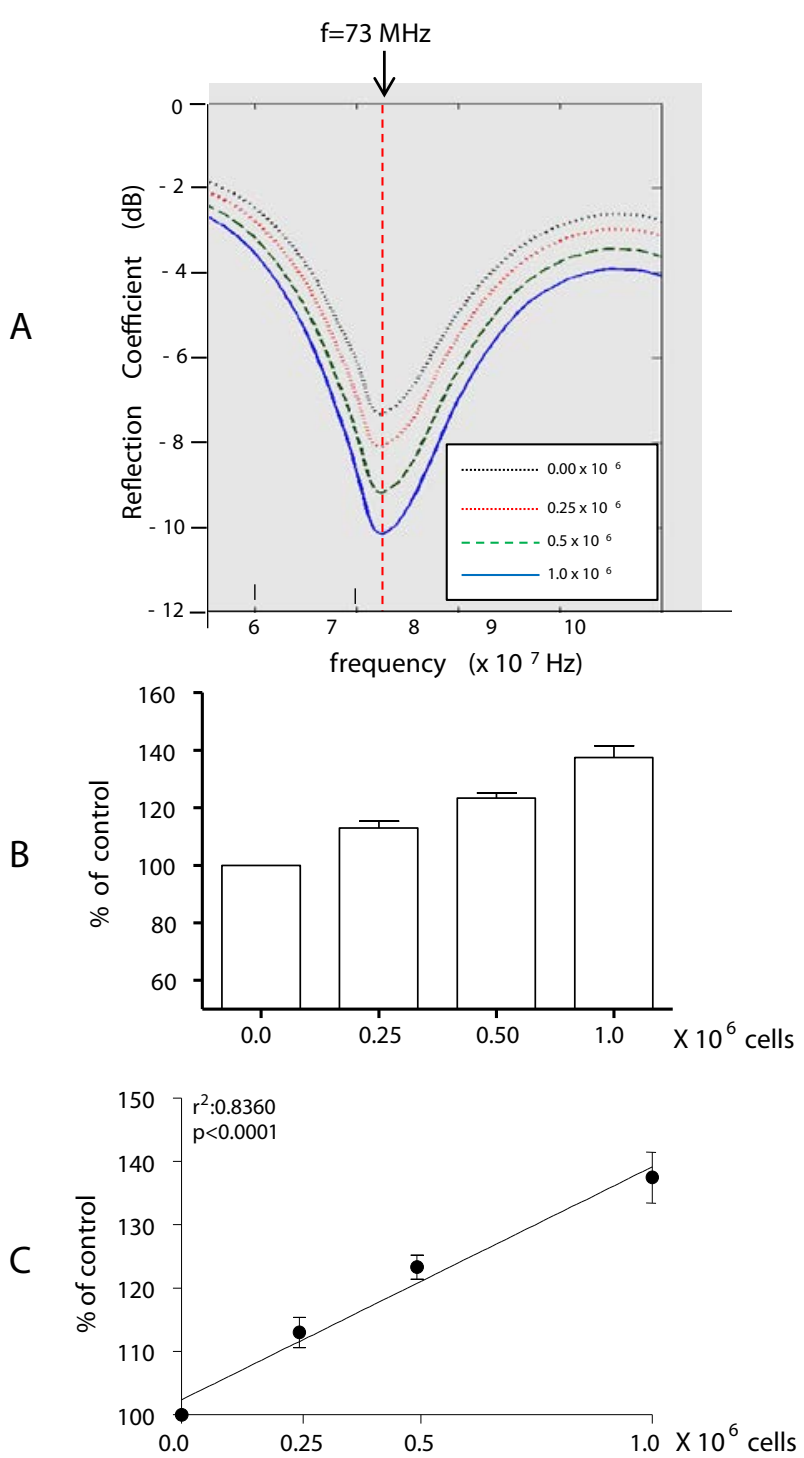

Figure 3: Determination of cell seeding in 3D liver cell model using reflection coefficient $\mathrm{S} 11(\mathrm{~dB}) .0,0.25 \times 10^{6}, 0.5 \times 10^{6}$ and $1 \times 10^{6}$ HepaRG cells are grown within scaffold for $24 \mathrm{~h}$. A) Reflection coefficient profiles S11 of 3D liver cell models consisting of different cell numbers. Plot of $\mathrm{S} 11(\mathrm{~dB})$ versus frequency $(\mathrm{HZ})$, measured with a network analyzer. Cell seeding was determined at frequency $\mathrm{f}=73 \mathrm{MHz}$. B) Data analysis of reflection coefficient S11 measurement at 73 $\mathrm{MHz}$, control (scaffold without cells) was set at $100 \%$, and C) Reference curve and linear regression, SEM $\pm 3-4$.

3D liver model. The external power density was held constant during the experiment. But the interacting dipoles and charges in the $3 \mathrm{D}$ liver model increased due to the increase of cell number within the scaffolds, because more ions are captured in cellular membrane compartments leading to higher ionic content and ionic mobility.

Thus, as the cell number increased in the 3D liver models, the number of interacting charges and dipoles increased and the intensity of the reflection coefficient S11 decreased (Figure 3A). As a consequence, due to this relationship precise recording and quantification of cell numbers in the PS- scaffolds is possible.

We figure that quantitative measurements of cell numbers in the PS-scaffolds can be determined through $\beta$-dispersion. We described the relationship between reflection coefficient $\mathrm{S} 11(\mathrm{~dB})$ and cell number to be at $73 \mathrm{MHz}$ in linear range $\left(\mathrm{p}<0.0001 ; \mathrm{r}^{2}=0.8360\right)$ (Figure $3 \mathrm{C})$. In a similar manner was the relationship between the absorption of blue formazan and the number of seeded cells which was determined using the biochemically method by means of MTT assay $(\mathrm{p}<0.0001$; $\mathrm{r} 2=0.9858)$ (Figure $4 \mathrm{C}$ ).

The S11-dependency on the cell number was used to setup a standard curve for the determination of cells. A linear calibration curve (Figure 3c) is applicable for the determination of the cell numbers in unknown 3D liver model samples.

The MTT assay is well established in characterizing cell proliferation in 2D monolayer cultures of low cell densities, but has the disadvantage of being invasive and destructive. It is a biochemical method to determine cell seeding, indirectly. It should be kept in mind that using the MTT method, dying or dead cells are not detected. But, prior to the extracting step with MTT lysis reagent, it gave information about the localization of the cell seeding within the PS-scaffold, because viable HepaRG cells were able to metabolize the yellow MTT reagent into blue formazan (Figure 4A) and the blue color localizes viable HepaRG cells of the 3D liver cell model. The more cells were seeded, the more blue color was located within the scaffolds (Figure 4A). The bar chart (Figure 4B) displays indirectly the increase of cell number

A
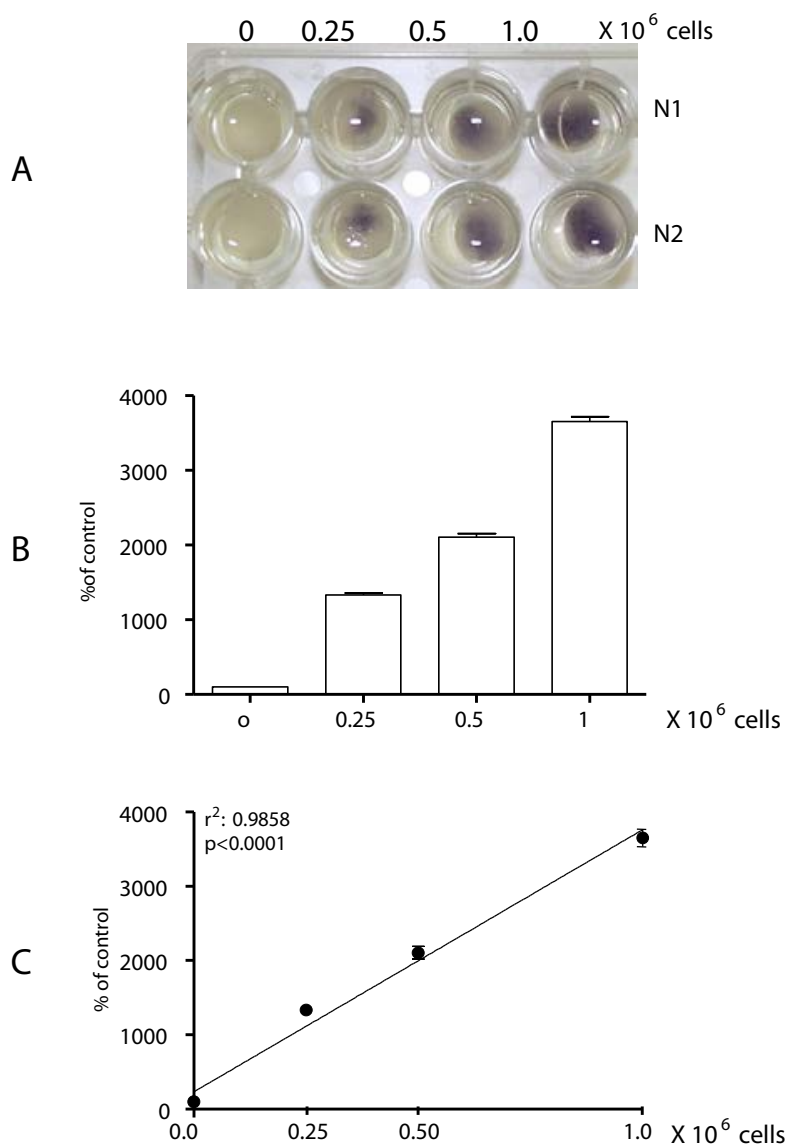

Figure 4: Determination of cell seeding in 3D liver cell model using MTT assay. $0,0.25 \times 10^{6}, 0.5 \times 10^{6}$ and $1 \times 10^{6}$ HepaRG cells are grown within scaffold for 24 h. A) Scaffolds in culture plates containing different numbers of HepaRG cells. The metabolic activity (MTT assay) of cells and cell seeding is analyzed optically via presence of intracellular blue formazan in the scaffolds. B) After adding cell lysis buffer and shaking, the blue formazan is extracted and MTT assay was analyzed colorimetrically, and C) Reference curve and linear regression, SEM $\pm 3-4$. 
after the colorimetrical analysis of the MTT assay after extraction of the blue color ((Figure 4B). In addition, the dependency of metabolic activity on the number of viable cells from the

$3 \mathrm{D}$ liver models were used to setup a reference curve for cell determination of unknown 3D samples (Figure 4c).

We have already tested the number of cells in scaffolds between 250.000-1.000.000 cells. These cell numbers are in a realistic range of cell culture effort to establish a $3 \mathrm{D}$ liver model. According the twostep time consuming cell culture protocol, the HepaRG cells need high cell density and special treatment to differentiate into hepatocyte-like cells [22]. But, using Bio-IS devices and electrodes that are appropriate adapted to the living system it is possible to detect less cell numbers in the scaffold. For instance, recent advances in microfabrication and labon-a- chip concept enabled Bio-IS-devices to detect and analyse a single cell. A single cell is placed inside an electric field that leads to a local distortion of the field enabling the measurement of the characteristic impedance signature of the cell [25].

The sensitivity of MTT assay depends on the cell type, their metabolic status and the appropriate solubilizing of the formazan crystals and was reported for a zebrafish liver cell line to led to low signals, at cell densities of $\leq 12.500$ cells/well [26].

\section{APAP effect on liver 3D cell model}

The toxic effect of APAP on the human liver 3D cell model was investigated. In our liver 3D cell model in vitro experiment is $5 \mathrm{mM}$ equivalent to $0.8 \mathrm{~g}$ APAP, $10 \mathrm{mM}$ APAP to $1.5 \mathrm{~g}$ and $20 \mathrm{mM}$ to $3.0 \mathrm{~g}$. The usual adult minimal daily dose of APAP is $1 \mathrm{~g}$, and the maximal daily dose is $4 \mathrm{~g}$ [27]. If the bioavailability of APAP is assumed to be $100 \%$, this means, that $1 \mathrm{~g}$ APAP is equivalent to $6.6 \mathrm{mM}$ and $4 \mathrm{~g}$ is equivalent to $26.5 \mathrm{mM}$ after intake to the human body. The cytotoxicity (LDH release from cells as a valid marker of membrane damage) increased with higher APAP dose ((Figure 6A) starting to differ significantly more than two fold compared to control from the concentration of $20 \mathrm{mM}$ APAP(Figure 6B). Consistently with the cytotoxicity was the dose-dependent behavior of the impedance, calculated from reflection coefficient S11 data after measuring at $73 \mathrm{MHz}$ (Figure 5). At the 3D liver cell model, S11 decreased and impedance at $73 \mathrm{MHz}$, as a percentage of the controls, increased significantly at $10 \mathrm{mM}$ APAP and above ( $\mathrm{p}<0.05$ vs. $0 \mathrm{mM}$ APAP=control). Figure 6 displays the dose-dependent APAP effect on cell viability in 3D liver cell model. In the case of the liver 3D model, the capacitance, or charge-storing characteristics were measured using Bio-IS and differ mostly at $73 \mathrm{MHz}$ after drug treatment. Supposing equal liver cell numbers (number of capacitors) at the beginning of the treatment with APAP, the ability of charging via Maxwell-Wagner polarization, which occurs because of the non-conductive nature of cellular membranes, decreased after drug exposure. The dose-dependent hepatotoxic drug effects of APAP on the liver 3D cell model, such as increasing loss of HepaRG cell membrane integrity and cellular ion content because cells were dying, may have changed the extracellular increased, the dielectric permittivity of the extracellular medium increased and the intensity of the reflection coefficient S11 decreased.

The measurement of more negative S11 values (lower reflection) coincided with decreasing metabolic changes, cell health and viability. It was reported that the frequency region around $100 \mathrm{MHz}$ could be caused by the dipole moment of large molecules, such as proteins [28]. Therefore, the dose-dependent liver cell response to APAP at $73 \mathrm{MHz}$ might also be caused by changes on the dipole moment of proteins.
A

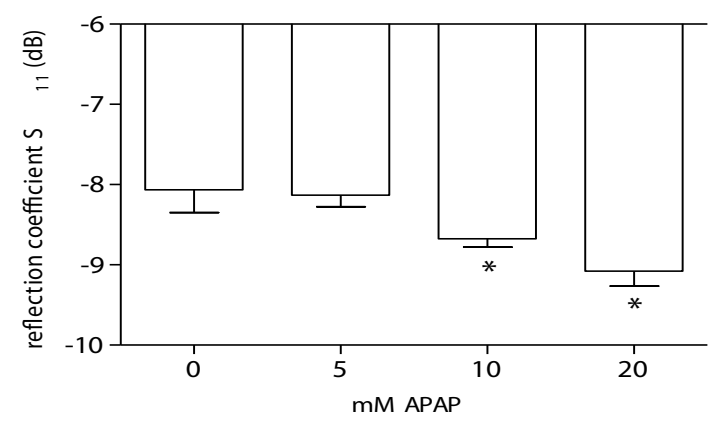

B

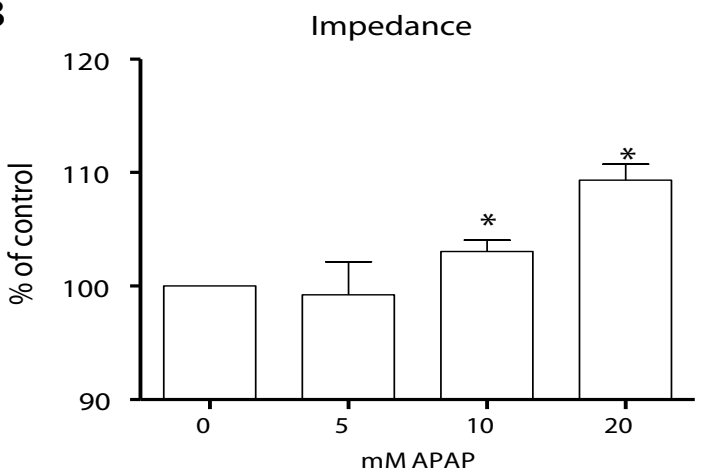

Figure 5: Dose-dependent effect of APAP on the 3D liver cell model analyzed using reflection coefficient S11 measurement by means of needle-like electrodes after $24 \mathrm{~h}$ incubation time with APAP. A) Bar chart of reflection coefficient S11. SEM $\pm 6-8$. ${ }^{*} p<0.05$ vs 0 mM APAP. B) Bar chart of calculated impedance at $73 \mathrm{MHz}, \mathrm{SEM} \pm 6-8 .{ }^{*}$ : $\mathrm{p}<0.05$ vs. $0 \mathrm{mM}$ APAP (untreated $3 \mathrm{D}$ liver cell models were set as $100 \%$ ).

During the manifestation of hepatotoxic effects, an increase in protein dysfunction is observed due to the accumulation of APAP protein adducts [29]. However, it should be kept in mind that APAP can have also intracellular effects that could influence charge of other organic macromolecules, such as other proteins, DNA, RNA or on intracellular membrane surrounded structures, like vesicles, mitochondria, or nuclei in liver cells [9], which are in the range of $\beta$-dispersion. Opposed to the increased cytotoxicity at the 3D liver cell model (Figure 6A), metabolic activity of the cells decreased with higher APAP doses (Figure 6B). Using the MTT assay, depletion of metabolic activity of HepaRG cells occurred already at a concentration of $5 \mathrm{mM}$ APAP and above $(\mathrm{p}<0.05$ vs. $0 \mathrm{mM}$ APAP (control). Therefore, the MTT test appeared to be most accurate, followed by S11-/Impedance technique, and subsequently the LDH assay.

The comparison of the three different techniques for analysis of the APAP effects on cell viability and cytotoxicity in the liver 3D model revealed that the effective concentration (EC) causing $15 \%$ loss of cell viability was about $5 \mathrm{mM}$ APAP for MTT assay (Figure 6B) and LDH assay (Figure 6A). Thus, the estimated EC10 values for both standard biochemical assays were below $5 \mathrm{mM}$ APAP. In contrast, the EC10 value was at a higher concentration at $20 \mathrm{mM}$ APAP for impedance analysis (Figure 5).

This implicates that the common biochemical assays were in case of APAP more sensitive than the new Bio-IS technique and our Bio-IS device needs further optimization. In summary, the label-free and nondestructive reflection coefficient S11 measurement and/or subsequently the determination of impedance might be suitable for monitoring drug 
A

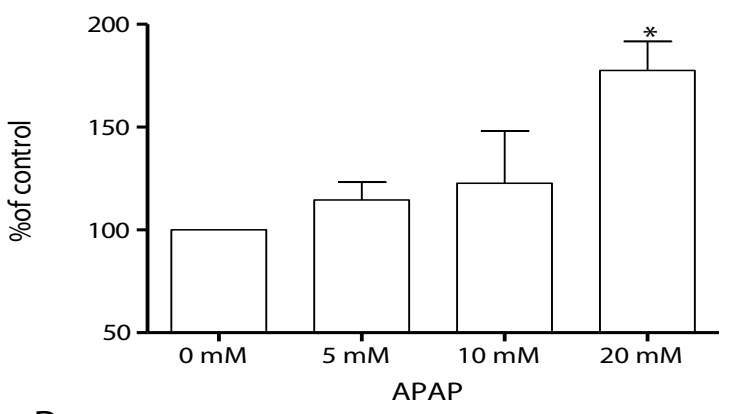

B

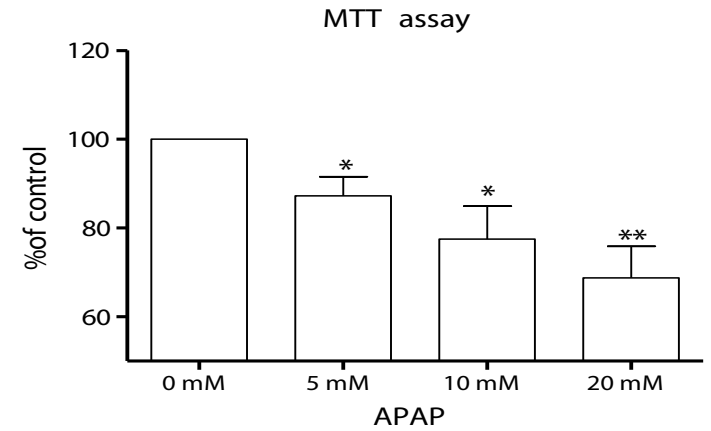

Figure 6: Dose-dependent effect of APAP on the 3D liver cell model analyzed using biological measurement techniques. A) Cell viability is determined by LDH assay and B) Metabolic activity is measured by MTT assay after $24 \mathrm{~h}$ incubation time with APAP. SEM $\pm 3-4 .{ }^{*} p<0.05$ vs 0 mM APAP and ${ }^{* *} p<0.005$ vs. 0 mM APAP (untreated 3D liver cell models were set as 100\%).

responses in 3D cell culture models under physiological conditions.

\section{Conclusion}

Electrical reflection coefficient S11 measurement is a new tool for advanced cell culture applications. Our Bio-IS test apparatus has many practical advantages over the international validated biochemical tests, that are gold-standard and international validated, such as MTT and $\mathrm{LDH}$ assay. One highlight is that it can measure the electrical passive properties of the 3D liver cell model for two different purposes, namely cell number determination and drug testing. Both purposes do have nothing in common at a first glance, but the change of the number of viable cells, differences in the amount of intact cellular membranes and ions that are separated in cellular compartments. Likely, the increase of the number of viable cells during cell proliferation and the decrease of the number of viable cells during drug testing at the $3 \mathrm{D}$ liver cell model was measured at one distinct frequency $(73 \mathrm{MHz})$.

Furthermore, it is non-destructive, requires low cost instrumentation, easy to applicable in practice and it gives ability of repeated assaying and on-line monitoring. Compared to the biochemical tests the used Bio-IS system is proposed to have broader applications and is not limited to cell viability/cytotoxicity testing. MTT and LDH assay are colorimetric tests, and interference with the test substance may occur [30-32].

The MTT test is an endpoint assay, while using LDH assay more than one measurement can be performed per experiment. Many methods of analysis exist for assaying for cell viability, cytotoxicity and other cellular responses (see elsewhere) and only few are here discussed. To overcome the two step procedure of the MTT assay, incubation with the MTT reagent and thereafter solubilization of formazan, the colorimetric MTS (3-(4,5-dimethylthiazol-2- yl)-5-(3carboxymethoxyphenyl)-2-(4-sulfo- phenyl)-2Htetrazolium) in vitro assay is an updated version of the international validated MTT method, MTS assay has the advantage of being soluble and no solubilisation step is required. 4-[3-(4-iodophenyl)-2-(4-nitro- phenyl)-2H-5-tetrazolio]1,3-benzene disul- fonate, is a water-soluble tetrazolium salt (WST-1) more stable, has broader linear range and enables more rapid color development [33].

Recently more and more used are Alamar Blue reagents, which are nontoxic, resazurin based, membrane permeable solutions that upon reduction form resorufin, a red fluorescent compound which can be quantitatively measured to determine viability [34]. Alamar Blue may circumvent many of the incompatibility issues described and offers many advantages over tetrazolium salts. To select an appropriate assay, different parameters like test substance, detection mechanism, specificity, and sensitivity have to be considered. Furthermore, the kind of biological material can influence test performance.

In summary, the evaluations obtained from disc-electrode measurements did not in practice produce satisfactory results and were therefore not appropriate. The disc-electrodes are bigger electrode structures, average over a bigger sample volume, but they may also sense much more parasitic current paths through the cell medium beyond the cells. Smaller electrodes, such as the needle-like electrodes, are more sensitive to changes in cell behavior in a 3D liver cell model, because they record from a more confined sample volume.

In addition, cell seeding and effects of drugs or chemicals on a 3D human cell culture system can be measured and analyzed with the described setup. Extracting the relevant information from reflection coefficient profiles obtained from the needle-like electrode measurement is a challenge in case of biological systems of high complexity and dynamics, because they can be described by a high number of parameters. The treatment of 3D liver cell models with increasing APAP doses reduced reflection coefficient dose-dependently (Figure 5A). Coincidently, there was a reduction of metabolic activity after treatment with increasing APAP doses. Because the reflection coefficient at $73 \mathrm{MHz}$ involves the changes of transformation of energy and matter in the 3D liver cell model, this physical parameter may reflect the behavior of the 3D liver cell model due to the metabolic activity. As well as the reflection coefficient, refers the metabolic activity to the set of chemical reactions associated with ionic or charge transfers that maintain life in living systems. Future research is required to determine in detail which of the distinct intracellular changes in the 3D liver cell model has influence on the Bio-IS effects we measured.

\section{Statistical analysis}

Results are expressed as means \pm SEM unless stated otherwise. Statistical differences between the two groups were assessed with the two-tailed unpaired t-test. The significance level was set at $\alpha=5 \%$ for all comparisons.

\section{Acknowledgement}

This work was partly supported by the state of Upper Austria (BioMed-zet Life Science $\mathrm{GmbH}$ ) and by the Austrian Center for Competence in Mechatronics ACCM (Institute for Microelectronics and Microsensors). We thank Dr. Nickl from GWP (Zorneding, Germany) for providing the image of Figure 2A.

\section{References}

1. Cox-Reijven PL, Soeters PB (2000) Validation of bio-impedance spectroscopy: effects of degree of obesity and ways of calculating volumes from measured resistance values. Int. J Obes. Relat Metab Disord. 24:271-280. 
Citation: Lornejad-Schäfer MR, Hilber W, Schäfer C (2014) Reflection Coefficient S11 Related Measurement System for Label-Free Cell Seeding Analysis and Drug Testing in a Three-Dimensional (3D) Cell Culture Model. J Biosens Bioelectron 5: 151. doi: 10.4172/2155-6210.1000151

2. Hope TA, lles SE (2004) Technology review: the use of electrical impedance scanning in the detection of breast cancer. Breast Cancer Res 6: 69-74.

3. Varlet-Marie E, Aloulou I, Mercier J, Brun JF (2010) Prediction of hematocrit and red cell deformability with whole body biological impedance. Clin Hemorheol Microcirc 44: 237-244.

4. Wegener J, Keese CR, Giaever I (2000) Electric cell-substrate impedance sensing (ECIS) as a noninvasive means to monitor the kinetics of cell spreading to artificial surfaces. Exp Cell Res 259: 158-166.

5. Verdonk E, Johnson K, McGuinness R, Leung G, Chen YW, et al. (2006) Cellular dielectric spectroscopy: a label-free comprehensive platform for functional evaluation of endogenous receptors. Assay Drug Dev Technol 4: $609-619$.

6. Yu N, Atienza JM, Bernard J, Blanc S, Zhu J, et al (2006) Real-time monitoring of morphological changes in living cells by electronic cell sensor arrays: an approach to study $\mathrm{G}$ protein-coupled receptors. Anal Chem 78: 35-43.

7. Guan JG, Miao YQ, Zhang QJ (2004) Impedimetric biosensors. J Biosci Bioeng 97: 219-226.

8. Ng KW, Leong DT, Hutmacher DW (2005) The challenge to measure cell proliferation in two and three dimensions. Tissue Eng 11: 182-191.

9. Hinson JA, Roberts DW, James LP (2010) Mechanisms of acetaminopheninduced liver necrosis. Handb. Exp. Pharmacol.369-405.

10. Yamada KM, Cukierman E (2007) Modeling tissue morphogenesis and cancer in 3D. Cell 130: 601-610.

11. Dobroschke M, Geldmacher Y, Ott I, Harlos M, Kater L, et al.(2009) Cytotoxic rhodium(III) and iridium(III) polypyridyl complexes: structure-activity relationships, antileukemic activity, and apoptosis induction. ChemMedChem. 4:177-187.

12. Bera UK, Nagaraju J (2011) Electricallmpedance Spectroscopic Studies on Broiler Chicken Tissue Suitable for the Development of Practical Phantoms in Multifrequency EIT. J Electr Bioimp 2:48-63.

13. Bashir R (2004) BioMEMS: state-of-the-art in detection, opportunities and prospects. Adv Drug Deliv Rev 56: 1565-1586

14. Hilber W, Lornejad-Schäfer MR, Schäfer C, Lederer T, Schröder K (2011) Impedance spectroscopy of a human hepatic $3 \mathrm{D}$ cell model during drug treatment: A comparative study with differently shaped electrodes. Procedia Engineering 25:1000-1003.

15. Kyle AH, Chan CT, Minchinton Al (1999) Characterization of three-dimensiona tissue cultures using electrical impedance spectroscopy. Biophys J 76: 26402648

16. Schäfer C, Lornejad-Schäfer MR, Schröder KR (2011) A human hepatic 3D-cel culture model for basic research, preclinical drug metabolism and toxicity testing. 2-74.

17. Maltman DJ, Przyborski SA (2010) Developments in three-dimensional cell culture technology aimed at improving the accuracy of in vitro analyses. Biochem Soc Trans 38: 1072-1075.

18. Mosmann T (1983) Rapid colorimetric assay for cellular growth and survival: application to proliferation and cytotoxicity assays. J Immunol Methods 65: 55-63.
19. Vendelin GD, Pavio AM, Rohde UL (2005) Microwave Circuit Design Using Linear and Nonlinear Techniques. Wiley-VCH.

20. Schwan HP (2011) Electrical properties of tissues and cell suspensions: mechanisms and models. 70-71.

21. Anthérieu S, Chesné C, Li R, Camus S, LahozA, et al. (2010) Stable expression, activity, and inducibility of cytochromes P450 in differentiated HepaRG cells. Drug Metab Dispos 38: 516-525.

22. Guillouzo A, Corlu A, AninatC, Glaise D, Morel F, (2007) The human hepatoma HepaRG cells: a highly differentiated model for studies of liver metabolism and toxicity of xenobiotics. Chem. Biol. Interact. 168:66-73.

23. Martinsen OG, Grimnes S, Schwan HP (2002) Interface phenomena and dielectric properties of biological tissue. 2643-2653.

24. Dean DA, Ramanathan T, Machado D, Sundararajan R (2008) Electrical Impedance Spectroscopy Study of Biological Tissues. J Electrostat 66: 165-177.

25. Giseok K, Yoo SK, Hyoung-IhIK, Jong-Hyun L (2011) Differentiation between Normal and Cancerous Cells at the Single Cell Level Using 3-D Electrode Electrical Impedance Spectroscopy. 1084-1089.

26. Bopp SK, Lettieri T (2008) Comparison of four different colorimetric and fluorometric cytotoxicity assays in a zebrafish liver cell line. BMC Pharmaco 8: 8

27. Schafer C, Schroder KR, Hoglinger O, Tollabimazraehno S, Lornejad-Schafer MR (2013) Acetaminophen changes intestinal epithelial cell membrane properties, subsequently affecting absorption processes. Cell Physiol Biochem. 32:431-447

28. Pethig R (1984) Dielectric Properties of Biological Materials: Biophysical and Medical Applications. Electrical Insulation, IEEE Transactions on El 19:453-474.

29. McGill MR, Yan HM, Ramachandran A, Murray GJ, Rollins DE, et al. (2011) HepaRG cells: a human model to study mechanisms of acetaminophen hepatotoxicity. Hepatology 53: 974-982.

30. Chakrabarti R, Kundu S, Kumar S, Chakrabarti R (2000) Vitamin A as an enzyme that catalyzes the reduction of MTT to formazan by vitamin C. J Cell Biochem 80: 133-138.

31. Talorete TP, Bouaziz M, Sayadi S, Isoda $H$ (2006) Influence of medium type and serum on MTT reduction by flavonoids in the absence of cells. Cytotechnology 52: $189-198$

32. Holder AL, Goth-Goldstein R, Lucas D, Koshland CP (2012) Particle-induced artifacts in the MTT and LDH viability assays. Chem Res Toxicol 25: 1885-1892.

33. Ngamwongsatit P, Banada PP, Panbangred W, Bhunia AK (2008) WST-1based cell cytotoxicity assay as a substitute for MTT-based assay for rapid detection of toxigenic Bacillus species using $\mathrm{CHO}$ cell line. J Microbiol Methods 73: $211-215$

34. Rampersad SN (2012) Multiple applications of Alamar Blue as an indicator of metabolic function and cellular health in cell viability bioassays. Sensors (Basel) 12: 12347-12360. 